\title{
CLINICAL AND RADIOGRAPHIC EVALUATION OF ACEMANNAN AS DIRECT PULP CAPPING MATERIAL IN YOUNG PERMANENT TEETH
}

\author{
Ahmed M.E. Alhag Ali*, Samy Youssef El-Bayoumy**, Abd-Elhamid Abu-Elyazed Eisa***
}

\begin{abstract}
Objective: The objective of this study was conducted to evaluate clinically and radiographically the effectiveness of acemannan as direct pulp capping material in young permanent teeth. Subjects and Methods: The present study was performed on thirty six mandibular first young permanent molars with deep carious lesion indicated for direct pulp capping. The children aged from six to nine years old. After completely removing the infected dentine, teeth with a pinpoint pulpal exposure were randomly divided into two treatment groups: (group A) Acemannan or (group B) calcium hydroxide paste. In both groups, the remaining part of the cavity was filled with resin-modified glass ionomer filling. The teeth were assessed clinically and radiographically at different intervals (48 hours, 1.5 month and 3 months post-operative). Results: The present study revealed that, the clinical and radiographic success rate of acemannan was (77.8\%), while Calcium hydroxide group showed a higher success rate (83.3\%). Conclusion: Although the acemannan is a promising material but calcium hydroxide offers good clinical and radiographic results and success than acemannan but with no statistically significant differences.
\end{abstract}

KEYWORDS: First young permanent molars, Direct pulp capping, Acemannan, Calcium hydroxide.

\section{INTRODUCTION}

Dental caries is a global health problem, the incidence of dental caries is higher in younger age groups; $60 \%-90 \%$ of school children suffers from dental caries ${ }^{(1)}$. Dental caries is one of the greatest challenges to the integrity of the developing tooth. It can result in irreversible pulpal damage, eventually causing necrosis of the pulpal tissues and associated arrested development of the tooth root. Ultimately, abnormal root development will impact the long-term prognosis for tooth retention (2). So direct pulp capping (DPC) may be treatment of choice in case of small pulp exposure to preserve pulp vitality to avoid or retard root canal treatment in an immature permanent molar exhibiting wide open apex, to allow continued root development, to allow odontoblasts to create dentin bridge between the pulp and the DPC materials, and to maintain pulp function ${ }^{(3)}$.

Direct pulp capping in teeth with incomplete root formation promote normal development of the root complex. There are long-term prognostic advantages of this treatment outcome over apexification treatment. The tooth structure formed is of a great quantity, and its composition appears to have greater structural integrity. The result is the fully developed tooth is more resistant to vertical root fracture ${ }^{(2,4)}$.

\footnotetext{
* Dentist, Medical Administration, Banha University.

** Professor, Department of Pedodontics and Oral Health, Faculty of Dental Medicine, Boys, Cairo, Al-Azhar University

*** Lecturer of Pedodontics and Oral Health, Faculty of Dental Medicine, Boys, Cairo, Al- Azhar University.
}

- Corresponding author: dr.ahmed.m.elhag@gmail.com

DOI: $10.21608 /$ ajdsm.2020.32294.1066 
Direct pulp capping involving the application of a medicament, dressing, or dental material to the exposed pulp in an attempt to preserve its vitality and to promote reparative dentine formation, i.e., a dentine bridge, and to seal the pulp exposure represents a preferable choice of treatment ${ }^{(5)}$. Ideally, a material for vital pulp procedures should biologically stimulate odontoblast/dental pulp cells and induce the formation of new hard tissue over the exposed pulp ${ }^{(6)}$.

In the literature, various materials have been suggested for DPC such as calcium hydroxide, Mineral trioxide aggregate (MTA), Biodentine, stem cells, propolis, novel endodontic cement, Emdogain, and TheraCal ${ }^{(7)}$. Among these materials utilized for pulp capping in young permanent teeth, calcium hydroxide $\left(\mathrm{Ca}(\mathrm{OH})_{2}\right)$ has long been the material of choice ${ }^{(8)}$.

Calcium Hydroxide has been considered the "gold standard" of direct pulp capping materials for several decades ${ }^{(9)}$. There are a number of advantages to calcium Hydroxide that have caused it to receive this recognition such as calcium hydroxide has high $\mathrm{pH}$ and excellent antibacterial properties ${ }^{(10)}$. The calcium Hydroxide has a long-term track record of clinical success as a direct pulp-capping agent in periods of up to 10 years ${ }^{(11)}$. The presence of tunnels in dentin barrier, extensive dentin formation obliterating the pulp chamber, high solubility in oral fluids, and lack of adhesion and degradation are some of the limitations reported with calcium hydroxide $^{(12,13)}$.

Because of these disadvantages of calcium hydroxide, Acemannan was suggested as an efficient and effective direct pulp capping material as it possesses potent anti-inflammatory and antimicrobial properties. This natural material demonstrated its biocompatibility and enhanced reparative dentin formation. Acemannan ( $a \beta$ $(1,4)$ acetylated polymannose ) extracted from Aloe Vera gel, showed cytocompatibility with various cell types ${ }^{(14,15)}$. These studies also showed that acemannan stimulated the proliferation, differentiation and mineralization of human dental pulp cells in permanent teeth. Moreover, when used as a DPC agent, acemannan enhanced reparative dentine formation in an animal model ${ }^{(15)}$.

\section{SUBJECTS AND METHODS}

The present study was carried out on thirty six mandibular first young permanent molars with deep carious lesion indicated for direct pulp capping. Patients age ranged from 6 - 9 years old. The child patients were selected from outpatient clinic of the department of Pedodontics and Oral health, Faculty of Dental medicine, Boys, Cairo, AlAzher University. The study was approved by the pedodontics scientific Committee and department council, Faculty of Dental Medicine, Boys, Cairo, Al-Azhar University

A power calculation indicates that we needed 18 teeth in each group (acemannan group and calcium hydroxide past group) to demonstrate the effect at $80 \%$ power in relation to the formula to examine the correlation with significance level 95\% (Z $\alpha=$ $1,96)$ and power test $80 \%(Z \beta=0,84)^{(16)}$.

The clinical inclusion criteria were as follows:

- All children (age from 6 to 9 years) with vital young first permanent molar tooth on testing by vitality test, such as thermal or electerical pulp tester.

- Cooperative child and parent.

- Presence of a deep carious lesion.

- Sufficient tooth structure for restoration.

- Absence of clinical signs or symptoms suggesting non-vital tooth such as spontaneous pain, tenderness to percussion, abscess, fistula, periodontal tissue swelling, or abnormal tooth mobility.

The radiographic criteria included, a deep carious lesion in close proximity to the pulp with an intact lamina dura. Absence of radiolucency at 
the furcation and periapical region, obliteration of the pulp and root canal, or internal/external root resorption.

\section{Exclusion criteria:}

- Patient with systemic diseases such as uncontrolled diabetic, blood diseases, or patient recive chemotherapy and radiotherapy.

- History of spontaneous, unprovoked toothache or mobility, (grade I, II and III).

- Sensitivity for percussion, no external or internal root resorption, no periapical lesions nor the presence of a fistulae.

After a complete history questionnaire and parental consent form were completed, hard and soft tissues were examined, the procedures completely explained to the parents as well as the children. Preoperative preapical $x$-ray was done for evaluation according to ADA instructions ${ }^{(17)}$. Molars to be treated were locally anaesthetized using mepecaine-L (a local anesthetic solution containing $20 \mathrm{mg}$ Mepivacaine hydrochloride with $0.06 \mathrm{mg}$ Levonordefrin hydrochloride) by a single block injection. A rubber dam was used to isolate the designated tooth (Ivory system). Cavity outline preparation was performed and all the unsupported enamel was removed mechanically by sterile ultrahigh speed round bur with irrigation then manually with a sharp sterile caries removal spoon excavator for excavation of soft carious dentine, all the carious dentin was removed ${ }^{(18)}$. The cavity was rinsed with sodium hypochlorite to decrease the bacterial load. Caries removal continued until the dentin offered resistance to hand excavation with the sharp dental spoon. Once the pulp tissue was exposed, hemostasis was achieved by applying pressure over the exposed pulp with cotton pellets that had been soaked with sterile saline solution for up to 10 minutes. If bleeding persisted after this procedure, the tooth was not included in the study. At this point, the tooth was allocated randomly to one of the two experimental groups:
Group A: Acemannan was mixed gently with distilled water and gently placed on the pulp exposure site ${ }^{(19)}$, care was taken to avoiding any pressure during placement of the material.

Group B: Calcium hydroxide (control group): According to manufacture instructions (Dentsply), an equal length of base and catalyst pastes was mixed till a desired thickness was obtained, then applied carefully on the pulp exposure site. Resinmodified glass ionomer filling was used as final restoration in both groups.

Clinical and radiographic [standardized periapical x-ray films] data of the two groups were collected and tabulated at the definite intervals (preoperative, 24 hours post-operative, 1.5 month and 3 months), and prepared for statistical analysis.

The significant differences between any two groups were assessed by Student's t-test. The differences between two groups were assessed by oneway analysis of variance (ANOVA) followed by the Fisher's exact test. These analyses were done using SPSS 23.0 statistical software.

\section{RESULTS}

Clinical and radiographic examinations were done preoperative, 24hours, 1.5 month and 3 months post-treatment. Patients' assessments were done in every visit and the success rate was regularly calculated. The final outcome was taken after 3 monthes.

\section{Clinical and Radiographic evaluation:}

In clinical evaluation patients' assessments were done in every visit to evaluate if there is any pain or swelling. In radiographic evaluation patients' assessments were done in every visit to evaluate if there is any widening of periodontal membrane space or periapical radiolucent areas.

\section{Clinical and radiographic findings of group A:}

After data chollection at the end of three months it was found that 14 teeth $(77.8 \%)$ responded positively 


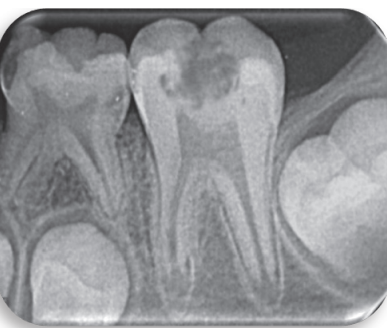

A: Preoperative

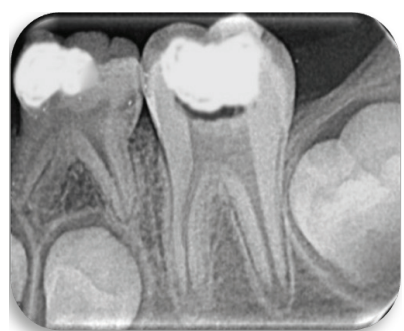

B: After 48 hours

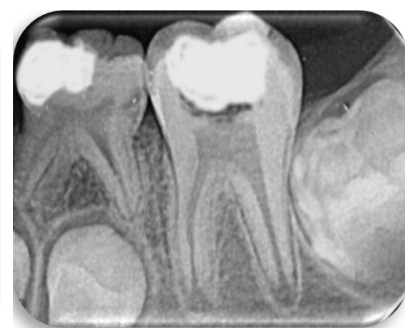

C: After 1.5 month

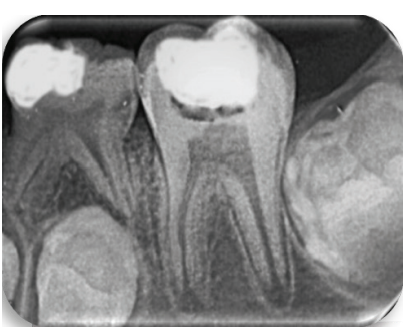

D: After 3 months

Fig (1a): (A,B,C,D) Acemannan direct pulp capping for first young permanent molar of an 8 years old girl (group A).

to acemannan material and appear normal clinically and radiographically after 48 hours, 1.5 month and 3 months. While the remaining 4 teeth (22.2\%) failed to respond positively, after 1.5 month 2 teeth had pain with percussion and swelling clinically, and radiographically showed widening of periodontal membrane space and periapical radiolucency. After 3 monthes another 2 teeth had pain with percussion and widening of periodontal membrane space, one of them had swelling and presence of periapical radiolucency. For this reason, Apexification and/or endodontic treatment were performed for them.

\section{Clinical and radiographic findings of group B:}

After data chollection at the end of three months it was found that 15 teeth $(83.3 \%)$ responded positively to calcium hydroxide and appear normal clinically and radiographically after 48 hours, 1.5 month and 3 months. While the remaining 3 teeth (16.7\%) failed to respond positively, after 1.5 month 2 teeth clinically had pain with percussion, one of them had swelling, and radiographically showed periapical radiolucency. One of them showed widening of periodontal membrane space. After 3 monthes another tooth had pain and swelling clinically and radiographically showed widening of periodontal membrane space and periapical radiolucency. For this reason, Apexification and/or endodontic treatment were performed for them.

\section{Comparison between two groups:}

On comparing the success rate of the groups, A, $B$, there was no statistically significant difference between Acemannan and Calcium hydroxide groups where $(\mathrm{p}=0.678)$. $($ Table 1$)$

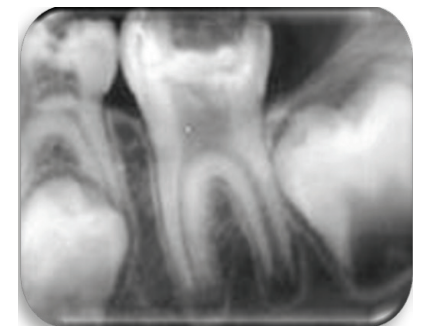

A: Preoperative

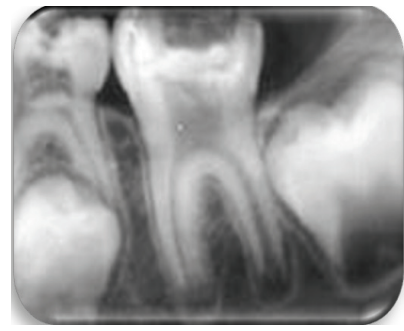

B: After 48 hours

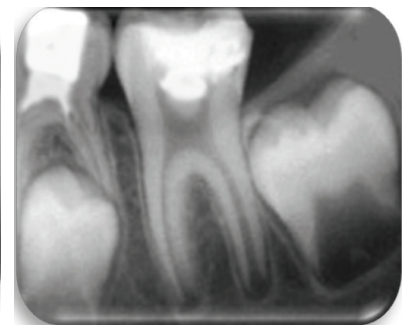

C: After 1.5 month

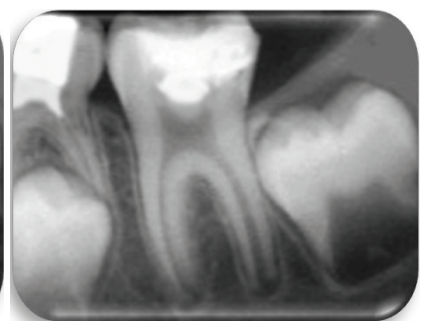

D: After 3 months

Fig (1b):(A,B,C,D) Calcium Hydroxide direct pulp capping for first young permanent molar of an 8 years old boy (group B). 
TABLE (1) The Success rate of both groups.

\begin{tabular}{|c|c|c|c|}
\hline \multicolumn{2}{|c|}{ Variables } & \multicolumn{2}{c|}{ Success rate } \\
\cline { 3 - 4 } Acemannan & Success & 14 & $77.8 \%$ \\
\cline { 2 - 4 } & Failure & 4 & $22.2 \%$ \\
\hline \multirow{2}{*}{ Calcium hydroxide } & Success & 15 & $83.3 \%$ \\
\cline { 2 - 4 } & Failure & 3 & $16.7 \%$ \\
\hline \multicolumn{2}{|c|}{$p$-value } & & \multicolumn{2}{|c|}{$\mathbf{0 . 6 7 8 n s}$} \\
\hline
\end{tabular}

*; ns; non-significant $(p>0.05)$

\section{DISCUSSION}

In the present study clinically and radiographically evaluated the effectiveness of acemannan as a direct pulp-capping (DPC) agent in mandibular first young permanent molar teeth with deep carious lesions when compared with calcium hydroxide paste. We found that the success rate in acemannan group was $(77.8 \%)$.

In a study done by Songsiripradubboon et al. ${ }^{(16)}$ investigated the clinical, radiographic, and histologic effects of acemannan used as direct pulp capping in human primary teeth for 6 months. They found that the success rate of Acemannan as direct pulp capping was $72.73 \%$ which approximately agrees with our study.

Another study done by Gonna et al. ${ }^{(19)}$ to compared the histopathological effects of acemannan to that of formocresol as pulp-dressing agents in primary teeth that underwent pulpotomy. The success rate of Acemannan and Formocresol groups was $(75 \%)$ and $(25 \%)$ respectively.

Songsiripradubboon et al. ${ }^{(20)}$, evaluated clinically and histologically of acemannan as a vital pulp therapy. Their results were $33 \%$ and $67 \%$ success rates for almost incomplete and complete mineralized bridge formation, respectively with normal pulp tissue without inflammation or pulp necrosis.
An in vitro study done by Jittapiromsak et al. ${ }^{(21)}$ showed that acemannan induced dental pulp cell proliferation, promoted differentiation of human primary dental pulp cells into osteoblast-like, cells extracellular matrix and growth factor synthesis, and new dentine formation as well as mineral deposition when used as a DPC agent.

The success rate of acemannan group may be due to its biocompatibility, potent anti-inflammatory and antimicrobial properties, which enhance reparative dentin formation. Treatment of human papillary cells by acemannan can lead to proliferation of dental pulp cells, activation of alkaline phosphatase, type I collagen, BMP-2, BMP-4, vascular endothelial growth factor, dentin saliva protein expression and mineralization ${ }^{(20-22)}$.

The faliur rate of acemannan may be due to extreme heat produced during cutting of dentin, presence of chronic inflammation in the coronal pulp or microleakage and poor seal ${ }^{(23)}$.

In calcium hydroxide group it was found that the success rate was (83.7\%). This result may be due to advantages of calcium hydroxide that has excellent antibacterial properties ${ }^{(24)}$, which are derived from several factors, included release of hydroxyl ions, which alkalise the environment $(\mathrm{pH}=11)$, that was not conducive for bacterial growth. Development of superficial necrosis and minor inflammation develops under the necrotic layer, which stimulates the pulp to defense, i.e. odontoblast differentiation and the formation of dentin bridge ${ }^{(25,26)}$. So in the present study, $\mathrm{Ca}(\mathrm{OH})_{2}$ was selected as a positive control group, which considered the "gold standard" of direct pulp capping materials for several decades ${ }^{(27)}$.

In a study done by Ulusoy et al. ${ }^{(28)}$ for 12 months, they found that the success rate of calcium hydroxide was $81.25 \%$ which approximately agrees with our study.

Another study done by Horsted et al. ${ }^{(29)}$ evaluated the survival rate of pulp capped teeth for 1, 3, 6 and 
12 months. It was found that the success rate of calcium hydroxide as direct pulp capping material was $82 \%$. which was similar with our study.

A randomized clinical trial done by Brizuela et al. ${ }^{(30)}$ compared the efficacy of calcium hydroxide, MTA, and Biodentine as pulp capping materials in young permanent molars, follow-up examinations were performed at 1 week, 3, 6 months, and 1 year. The success rate of $\mathrm{Ca}(\mathrm{OH})_{2}$ was $(86.36 \%)$ which slightly higher than our study.

Lower success rate of direct pulp capping with calcium hydroxide has been showed in studies done by Iwamoto et al. ${ }^{(31)}$ and Cengiz et al. ${ }^{(32)}$ which was $78.3 \%$ and $73.3 \%$ respectively.

An explanation of $\mathrm{Ca}(\mathrm{OH})_{2}$ failure could be due to the disadvantages of calcium hydroxide, limitations and side effects. No inherent adhesive properties, provides a poor seal, self-cure formulations are soluble and subject to dissolution over time (33) pulpal inflammation, internal resorption, pulp canal obliteration, presence of tunnel defects and weakness of pulpal responses ${ }^{(34)}$. Also penetration of bacteria or presence of chronic inflammation in the exposure site at the time of treatment and as such leading to diagnostic errors.

\section{CONCLUSIONS}

Acemannan is a promising material that can be used as a pulp capping material with a good handling and showing competitive properties to calcium hydroxide. Calcium hydroxide offers better clinical and radiographic results when compared with acemannan as a direct pulp capping material.

\section{REFERENCES}

1. Abbass MMS, Mahmoud SA, El Moshy S, Rady D, AbuBakr N, Radwan IA, et al. The prevalence of dental caries among Egyptian children and adolescences and its association with age, socioeconomic status, dietary habits and other risk factors. A cross-sectional study. F1000Res. 2019;8:8
2. Fuks A, Kupietzky A, Guelmann M. Pulp Therapy for the Primary Dentition. Pediatric Dentistry 23. 6 ed. Philadelphia: Content Repository Only; 2019. p. 329-51.

3. Brizuela C, Ormeño A, Cabrera C, Cabezas R, Silva CI, Ramírez V, et al. Direct pulp capping with calcium hydroxide, mineral trioxide aggregate, and biodentine in permanent young teeth with caries: a randomized clinical trial. 2017;43(11):1776-80.

4. Anil M, Suvarna P, Upendra H, Girish U, Preshit P. Mineral Trioxide Aggregate Pulpotomy for Permanent Molars with Clinical Signs Indicative of Irreversible Pulpitis: A Case Report. Journal of Medical Science And clinical Research. 2018;6(5):835-41.

5. Guideline on pulp therapy for primary and young permanent teeth. Pediatr Dent. 2008;30(7 Suppl):170-4.

6. Ferracane J, Cooper P, Smith A. Can interaction of materials with the dentin-pulp complex contribute to dentin regeneration? Odontology. 2010;98(1):2-14.

7. Qureshi A, Soujanya E, Nandakumar P. Recent advances in pulp capping materials: an overview. Journal of clinical diagnostic research. 2014;8(1):316.

8. Olsson H, Petersson K, Rohlin M. Formation of a hard tissue barrier after pulp cappings in humans. A systematic review. International endodontic journal. 2006;39(6):42942.

9. Dubey A. Calcium hydroxide in dentistry: A Review. Chetinad Health City Medical Journal. 2016;5:30-3.

10. Chandrasekhar H, Sharma S, Research. Efficient Agents for Pulp Protection: A Review. Journal of Pharmaceutical Sciences. 2016;8(11):1308-11.

11. Auschill T, Arweiler N, Hellwig E, Zamani A, Sculean A. Success rate of direct pulp capping with calcium hydroxide. Schweizer Monatsschrift für Zahnmedizin. 2003;113:946-52.

12. Bollu I, Velagula L, Bolla N, Kumar K, Hari A, Thumu J. Histological evaluation of mineral trioxide aggregate and enamel matrix derivative combination in direct pulp capping: An in vivo study. Journal of conservative dentistry 2016;19(6):536-40.

13. Li Z, Cao L, Fan M, Xu Q. Direct pulp capping with calcium hydroxide or mineral trioxide aggregate: a metaanalysis. Journal of endodontics. 2015;41(9):1412-7.

14. Jettanacheawchankit S, Sasithanasate S, Sangvanich P, Banlunara W, Thunyakitpisal P. Acemannan stimulates gingival fibroblast proliferation; expressions of keratinocyte growth factor-1, vascular endothelial growth factor, 
and type I collagen; and wound healing. Journal of pharmacological sciences. 2009;109(4):525-31.

15. Sierra G, Castro R, Gonzalez A, Lara J, Chávez-Montes A. Acemannan, an extracted polysaccharide from Aloe vera: A literature review. Natural product communications. 2014;9(8):1217 - 21.

16. Songsiripradubboon S, Banlunara W, Sangvanich P, Trairatvorakul $\mathrm{C}$, Thunyakitpisal $\mathrm{P}$. Clinical, radiographic, and histologic analysis of the effects of acemannan used in direct pulp capping of human primary teeth: short-term outcomes. Odontology. 2016;104(3):329-37.

17. American Dental Association. Dental radiographic examinations: recommendations for patient selection and limiting radiation exposure. Columbia: American Dental Association. 2012

18. Kidd E, Ricketts D, Beighton D. Criteria for caries removal at the enamel-dentine junction: a clinical and microbiological study. British dental journal. 1996;180(8):287-91.

19. Gonna S, Deraz E, Ghoname N, Kabbash A, Yagi A. Acemannan and formocresol pulpotomies in primary teeth: a comparative histopathological study. Gastroenterology and Hepatology Research. 2017;6(4):2386-91.

20. Songsiripradubboon S, Kladkaew S, Trairatvorakul C, Sangvanich P, Soontornvipart K, Banlunara W, et al. Stimulation of Dentin Regeneration by Using Acemannan in Teeth with Lipopolysaccharide-induced Pulp Inflammation. Journal of Endodontics. 2017;43(7):1097-103.

21. Jittapiromsak N, Sahawat D, Banlunara W, Sangvanich P, Thunyakitpisal P. Acemannan, an Extracted Product from Aloe Vera , Stimulates Dental Pulp Cell Proliferation, Differentiation, Mineralization, and Dentin Formation. Tissue engineering Part A. 2010;16:1997-2006.

22. Boonyagul S, Banlunara W, Sangvanich P, Thunyakitpisal P. Effect of acemannan, an extracted polysaccharide from Aloe vera, on BMSCs proliferation, differentiation, extracellular matrix synthesis, mineralization, and bone formation in a tooth extraction model. Odontology. 2014;102(2):310-7.

23. Trope M. Root resorption due to dental trauma. Endodontic topics. 2002;1(1):79-100.

24. Barthel C, Levin L, Reisner H, Trope M. TNF- $\alpha$ release in monocytes after exposure to calcium hydroxide treated
Escherichia coli LPS. International Endodontic Journal. 1997;30(3):155-9.

25. Komabayashi T, Zhu Q, Eberhart R, Imai Y. Current status of direct pulp-capping materials for permanent teeth. Dental materials journal. 2016;35(1):3-10.

26. Ricucci D, Loghin S, Lin L, Spångberg L, Tay F. Is hard tissue formation in the dental pulp after the death of the primary odontoblasts a regenerative or a reparative process? Journal of dentistry. 2014;42(9):1156-70.

27. Akhlaghi N, Khademi A. Outcomes of vital pulp therapy in permanent teeth with different medicaments based on review of the literature. Dental research journal. 2015;12(5):406.

28. Ulusoy A, Bayrak S, Bodrumlu E. Clinical and radiological evaluation of calcium sulfate as direct pulp capping material in primary teeth. European Journal of Paediatric Dentistry. 2014;15(2):127-31.

29. Horsted P, Sondergaard B, Thylstrup A, El Attar K, Fejerskov $\mathrm{O}$. A retrospective study of direct pulp capping with calcium hydroxide compounds. Dental Traumatology. 1985;1(1):29-34.

30. Brizuela C, Ormeno A, Cabrera C, Cabezas R, Silva C, Ramirez V, et al. Direct Pulp Capping with Calcium Hydroxide, Mineral Trioxide Aggregate, and Biodentine in Permanent Young Teeth with Caries: A Randomized Clinical Trial. Journal of Endodontics. 2017;43(11):1776-80.

31. Iwamoto C, Adachi E, Pameijer C, Barnes D, Romberg E, Jefferies S. Clinical and histological evaluation of white ProRoot MTA in direct pulp capping. American Journal of Dentistry. 2006;19(2):85-90.

32. Cengiz E, Yilmaz H. Efficacy of Erbium, Chromiumdoped:Yttrium, Scandium, Gallium, and Garnet Laser Irradiation Combined with Resin-based Tricalcium Silicate and Calcium Hydroxide on Direct Pulp Capping: A Randomized Clinical Trial. Journal of Endodontics. 2016;42(3):351-5.

33. Hilton TJ. Keys to clinical success with pulp capping: a review of the literature. Journal of Operative dentistry 2009;34(5):615-25.

34. Morotomi T, Washio A, Kitamura C. Current and future options for dental pulp therapy. Japanese Dental Science Review. 2019;55(1):5-11. 